\title{
Identification of single nucleotide polymorphisms in FOXJ1 and their association with allergic rhinitis
}

Received: 6 October 2005/ Accepted: 8 December 2005/Published online: 4 March 2006

(C) The Japan Society of Human Genetics and Springer-Verlag 2006

\begin{abstract}
Forkhead-box J1 $(F O X J 1)$ is a presumed transcription factor that can suppress $\mathrm{T}$ cell activity, at least partially, through the repression of $N F \kappa B$ activity. Thus, dysregulation of FOXJ1 is thought to be associated with autoimmune diseases and/or other inflammatory diseases. To investigate the association between single nucleotide polymorphisms (SNPs) of human FOXJ1 and allergic rhinitis, we scanned the whole human FOXJ1 gene, including the promoter region, by direct sequencing of DNA from 32 individuals. We identified seven SNPs, three of which $(g .-460 C>T$, g. $1805 G>T$, and $g .3375 G>C$ ) were chosen for large sample size genotyping $(n=713)$, and to assess the genotype frequencies of these SNPs between controls and allergic rhinitis patients. We also investigated the relationships of each genotype with serum total IgE levels in allergic rhinitis patients, and compared the frequencies of haplotypes constructed by these SNPs between the two
\end{abstract}

Chun-Shi Li and Soo-Cheon Chae contributed equally to this work.

C.-S. Li $\cdot$ H.-T. Chung $(\bowtie)$

Department of Microbiology and Immunology,

School of Medicine, Wonkwang University, Iksan,

570-749 Chonbuk, South Korea

E-mail: htchung@wonkwang.ac.kr

Tel.: + 82-63-8506762

Fax: + 82-63-8515066

S.-C. Chae $\cdot$ H.-T. Chung

Genome Research Center for Immune Disorders,

School of Medicine, Wonkwang University, Iksan,

570-749 Chonbuk, South Korea

J.-H. Lee

Department of Otolaryngology, School of Medicine, Wonkwang University, Iksan, 570-749 Chonbuk, South Korea

C.-S. $\mathrm{Li}$

Department of Pharmacology, Yanbian University

Medical College, Yianji, 133000 Jilin, China

Q. Zhang

Department of Microbiology and Immunology, Yanbian University Medical College, Yianji, 133000 Jilin, China groups. Our results suggest that the SNPs $g .-460 C>T$, g. $1805 G>T$ and $g .3375 G>C$ in the human $F O X J 1$ gene might be associated with susceptibility to allergic rhinitis $(P=0.0184,0.0076$, and 0.0143 , respectively). The main haplotype, CGG, also revealed a significant association with allergic rhinitis $(P=0.000018)$. However, no significant association was found between serum total $\operatorname{IgE}$ levels and the genotypes of these polymorphisms.

Keywords $F O X J 1 \cdot$ Polymorphism ' Haplotype · Allergic rhinitis $\cdot \mathrm{T}$ cell

\section{Introduction}

Allergic rhinitis is a very common disease, affecting about $10-25 \%$ of the World's population, and the incidence has been increasing over the last few decades (Sibbald 1993; Lundback 1998). Genetic and environmental factors contribute to the occurrence of this disease (Cookson 2002). Allergic rhinitis is initiated by the meeting of atopic individuals with innocuous antigens, such as pollen, house dust, mites, and so on, followed by a series of cellular interactions, ultimately resulting in the inflammation of nasal mucosa, accompanied by elevation of serum IgE levels and recruitment of eosinophil to the site of allergic reactivity (Passali et al. 2001). T-helper type 2 (Th2) cells play a critical role in the development of this disease. Naïve $\mathrm{T}$ helper cells can differentiate into Th1 or Th2 subsets according to the antigen type, antigen delivery route, cytokine profile, transcription factors, and signaling pathways. Th1 cells produce cytokines such as IL-2, IFN- $\gamma$, TNF- $\beta$; Th2 cells produce cytokines like IL-4, IL-5, and IL-10 (Ho and Glimcher 2002). The balance between Th1 and Th2 is very important in maintaining the healthy state of the body. While predominance of Th1 cytokines is associated with autoimmune diseases, a predominance of $\mathrm{Th} 2$ cytokines may cause asthma and allergic diseases.

The forkhead-box (FOX) gene family encodes transcription factors containing a DNA-binding domain 
known as the forkhead domain (Weige and Jackle 1990). There are more than 100 members in this gene family. Humans have at least 43 members, which have been classified into 17 subclasses: FOXA to FOXQ (Kaestner et al. 2000). FOX family factors take part in diverse biological processes including development, metabolism, aging, and cancer (Carlsson and Mahlapuu 2002; Tran et al. 2003). Many members of this family play critical roles in the regulation of immune responses. For instance, Foxnl is associated with thymic epithelial cell development, and mutation of Foxn1 can cause severe immunodeficiency. Foxo proteins are important in the maintaining of quiescence and tolerance of $\mathrm{T}$ and $\mathrm{B}$ cells, and Foxp3 plays a critical role in regulatory $\mathrm{T}$ cell development (Coffer and Burgering 2004). Foxjl, a member of the forkhead family also known as HFH-4 and FKHL-13, is expressed mainly in the ciliated cells of lung, choroids plexus, and oviduct, and is linked with developmental regulation in ciliated epithelial cells and the determination of the left-right axis (Chen et al. 1998; Blatt et al. 1999; Brody et al. 2000; Okada et al. 2004; Lim et al. 1997). Recently, Lin et al. (2004) found that Foxj1-deficient mice suffered multi-organ systemic inflammation, exaggerated Th1 cytokine production, and $\mathrm{T}$ cell proliferation in autologous mixed lymphocyte reactions. While Foxj1 suppressed NF- $\kappa \mathrm{B}$ transcription activity, Foxj1-deficient T cells possessed increased NF$\kappa \mathrm{B}$ activity (Lin et al. 2004). Therefore, Foxjl is thought to be a potential candidate gene associated with autoimmune and/or other inflammatory diseases.

In this study, we identified single nucleotide polymorphisms (SNPs) in the human FOXJ1 gene by direct sequencing of genomic DNA isolated from 32 individuals, and analyzed the association of genotype and allele frequencies between allergic rhinitis patients and nonallergic rhinitis controls. We further investigated the relationships between these polymorphisms and $\operatorname{IgE}$ levels in allergic rhinitis patients, and calculated the haplotype frequencies constructed by these SNPs in both groups.

\section{Materials and methods}

\section{Patients and DNA samples}

Genomic DNA samples were obtained from 418 controls (260 males and 158 females) without allergic rhinitis and 295 allergic rhinitis patients (191 males and 104 females). The mean ages of controls and patients were 38.3 and 24.0 years, respectively. The allergic rhinitis patients were recruited from the outpatient clinic of Wonkwang University Hospital. The diagnosis was based on symptoms of sneezing, watery rhinorrhea, nasal obstruction, and the result of a positive skin test. The skin test was performed with six common aeroallergens from house dust mites, house dust, grass mix, tree pollens, shed animal hair, and molds (Torii; Tokyo, Japan). All of the patients with allergic rhinitis had a history of all of the symptoms and had a positive skin test. The controls were recruited from members of the general population who took a comprehensive medical examination at Wonkwang University Hospital. All subjects in this study were Korean and were living in the same area. Genomic DNA was extracted from leukocytes in peripheral blood by a standard phenol-chloroform method or by Puregene DNA Purification Kit (Gentra Systems, Minneapolis, MN) according to manufacturer's directions.

\section{Polymerase chain reaction}

The full length of FOXJI including promoter region $(\sim 2.2 \mathrm{~kb})$ were amplified by polymerase chain reaction (PCR) using the three primer pairs (FOXJ1-F1: 5'-TGTGCCATCTGTGTACCCCTCT-3' and FOXJ1R1: 5'-ACCCCTAAAGCCCTCCACCTCT-3', FOXJ1F2: $5^{\prime}$-CTCGCTCCCCACGCTGCACT- $3^{\prime}$ and FOXJ1-R2: 5'-GCCCTCCCTCACCCTGCCGCT-3', and FOXJ1F3: 5'-TCTCCACTCGCGGCTGCCCA-3' and FOXJ1-R3: 5'-CCCGCAGCTGCCGCTCAGCCA-3'). PCR was performed in a $20 \mu \mathrm{l}$ reaction volume and the reaction mixture was prepared according to the instructions with the EF-Taq protocol (Solgent, Korea). Amplification was carried out in a GeneAmp PCR system 9700 thermocycler (Applied Biosystems, Foster City, CA) at $95^{\circ} \mathrm{C}$ for $5 \mathrm{~min}$ to pre-denature the template DNA, followed by 30 cycles of denaturation at $98^{\circ} \mathrm{C}$ for $10 \mathrm{~s}$, annealing at $68^{\circ} \mathrm{C}$ for $30 \mathrm{~s}$, and extension at $72^{\circ} \mathrm{C}$ for $2.5 \mathrm{~min}$, with a final extension for another 10 min at $72^{\circ} \mathrm{C}$ to end the reaction. The PCR products were purified with a PCR Purification Kit (Millipore, Bedford, MA) and were used as template DNA for direct sequencing and single base extension (SBE) reactions.

\section{SNP discovery and SBE}

Identification of SNPs was carried out by direct sequencing. Besides the two forward primers, FOXJ1-F1 and FOXJ1-F3, which were used for PCR amplification, 12 primers were additionally used for DNA sequence analysis (Table 1). Sequence analysis was performed by Bigdye Terminator cycle sequencing using the ABI 3100 Prism Automated DNA sequencer (Applied Biosystems) according to the manufacturer's instructions. The reference sequence is based on the sequence of human chromosome 17, clone RP11-449J21 (AC018665). Three SNPs $(g .-460 C>T, g .1805 G>T$, and $g .3375 G>C$ ) out of seven SNPs identified in this study were selected for large samples genotyping based on locations and linkage disequilibrium (LD). Genotyping for large sample sizes was performed by the SBE method using the ABI prism SnaPshot Multiplex Kit (Applied Biosystems) as described previously (Chae et al. 2004, 2005a). The three SBE primers used in this study are shown in Table 1. 


\section{Statistical analysis}

The allergic rhinitis patients and controls were compared by case-control association analysis. The $\chi^{2}$ test was used to estimate the Hardy-Weinberg equilibrium (HWE). Allele frequency was defined as the percentage of individuals carrying the allele among the total number of individuals. Logistic regression analyses were used to calculate odds ratios ( $95 \%$ confidence interval) for SNP sites. LD analyses by pair-wise comparison of biallelic loci and the haplotype frequencies of $F O X J 1$ for multiple loci were estimated using the expectation maximization (EM) algorithm with SNPAlyze software (Dynacom, Mobara-shi, Japan). ANOVA was applied to analyze differences between genotypes, and the serum total IgE levels in allergic rhinitis patients. A $P$ value of less than 0.05 was considered to indicate statistical significance.

\section{Results and discussion}

Human FOXJ1, which is located on chromosome 17q25, is a member of the forkhead gene family and consists of two exons and one intron. To examine variation at sites in the FOXJ1 gene including the promoter region, we scanned genomic DNAs isolated from 32 unrelated controls by direct DNA sequencing. We identified a total of seven SNPs, g. $460 C>T(\mathrm{rs} 880213)$ and $g .-$ $342 G>C \quad(\mathrm{rs} 880212)$ in the promoter region, g.1164G $>C$, g. $1805 G>T$ (rs1868823), g.1824C $>G$ and g. $1849 G>C$ (rs1868824) in intron 1 , and $g .3375 G>C$ (rs3192453) in the $3^{\prime}$-UTR region (Fig. 1). LD coefficients $\left(\left|D^{\prime}\right|\right.$ and $\left.r^{2}\right)$ between all SNP pairs identified in this study were calculated (Table 2).

Among the polymorphisms identified in this study, three SNPs $(g .-460 C>T, g .1805 G>T$, and $g .3375 G>C$ ) were selected for large sample genotyping based on their location and LD. We analyzed the genotype of these SNPs in allergic rhinitis patients and in controls by the SBE method, and compared the genotype frequencies between these groups. The genotype frequencies of all loci were in HWE $(P>0.05$, data not shown). LD coefficients $\left(\left|D^{\prime}\right|\right)$ between all SNP pairs were calculated, and absolute LD $\left(\left|D^{\prime}\right|=1\right.$ and $\left.r^{2}=1\right)$ was found between $g .3375 G>C$ and $g .-460 C>T$, and g. $3375 G>C$ and $g .1805 G>T$ in allergic rhinitis patients (data not shown). The $P$ values of each polymorphism were analyzed with respect to comparison between allergic rhinitis patients and the controls (Table 3). The genotype and allele frequencies of $g$.$460 C>T$ in allergic rhinitis patients were significantly different from those in the control group $(P=0.0184$ and 0.0143 , respectively). Furthermore, the genotype frequencies of $g .1805 G>T$ as well as $g .3375 G>C$ between those groups are also significantly different $(P=0.0076$ and 0.0143 , respectively). These results strongly suggest that the SNPs of FOXJ1 might be associated with susceptibility to allergic rhinitis (Table 3). We further compared total serum IgE levels between each genotype of these polymorphisms in allergic rhinitis patients. These polymorphisms were shown to have no significant association with total serum IgE levels in allergic rhinitis patients (data not shown). This result implies that these polymorphisms might not affect $\mathrm{IgE}$ production in allergic rhinitis patients.

We calculated the haplotype frequencies among the g. $-460 C>T, g .1805 G>T$, and $g .3375 G>C$ SNPs of FOXJ1 between controls and allergic rhinitis patients. While eight haplotypes were identified with three major haplotypes, explaining more than $94.0 \%$ of the distribution in controls, four haplotypes in allergic rhinitis patients were identified with two major haplotypes $(96.9 \%)$ out of eight possible haplotypes (Table 4). These results showed that the haplotype frequencies between controls and allergic rhinitis patients were significantly different, and suggested that the FOXJI polymorphisms might be one of the most important genetic factors in allergic rhinitis susceptibility.

Allergic rhinitis is a multi-complex disorder influenced by genetic predisposition and environmental

Table 1 Primer sequences for DNA sequencing and genotyping of the forkhead-box J1 (FOXJ1) gene

\begin{tabular}{|c|c|c|c|}
\hline Application & Primer name & Primer sequence $\left(5^{\prime} \rightarrow 3^{\prime}\right)$ & Region \\
\hline \multirow{8}{*}{ DNA sequencing } & FOXJ1-SF1 & GACACAGTCCCCAGAGTCCCT & Promoter \\
\hline & FOXJ1-SF2 & CTGAGCCCGCTCCCAGCCT & Promoter \\
\hline & FOXJ1-SF4 & AGGGGGTCCTGATTTCCTGCTCT & Promoter \\
\hline & FOXJ1-SF5 & CGTGGATGCTGACCGCCCCCT & Promoter \\
\hline & FOXJ1-SF6 & TGСТСТСТСССТGCACCCA & Exon 2 \\
\hline & FOXJ1-SF10 & TGTCCCAGCCCCCCACCTCA & Intron 1 \\
\hline & FOXJ1-SF11 & AAAGCGGGCAGGCCACAGGA & Intron 1 \\
\hline & FOXJ1-SF12 & AGGTGGAGGGCTTTAGGGGT & Intron 1 \\
\hline \multirow[t]{3}{*}{ Single base extension (SBE) } & FOXJ1-SS1 & ACCGCCCTCCCAAACTTCTCGCCCCA & $g .-460 C>T$ \\
\hline & FOXJ1-SS2 & TCATCAGATCCCCCACCTCTCATCCCTGCGC & $g .1805 G>T$ \\
\hline & FOXJ1-SS3 & CCTGGAGGAGCCCGGGTCAG & $g .3375 G>C$ \\
\hline
\end{tabular}




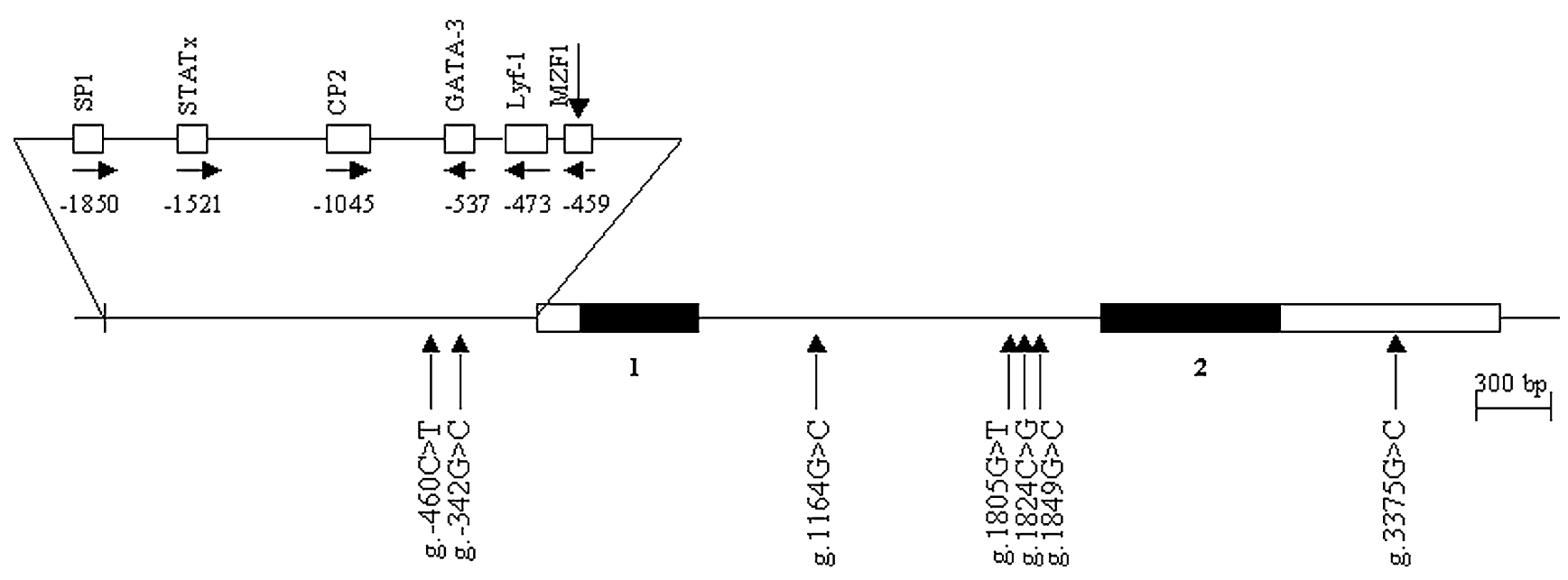

Fig. 1 Locations of each single nucleotide polymorphism (SNP) in the forkhead-box $\mathrm{J} 1$ gene (FOXJ1). Black blocks Coding exons, white blocks $5^{\prime}$ - and $3^{\prime}$-UTR. Positions of SNPs were calculated factor sites were searched at http://www.cbrc.jp/research/db/ TFSEARCH.html. The reference sequence is based on human relative to the translation start site. Putative transcription chromosome 17 clone RP11-449J21 (AC018665)

Table 2 Linkage disequilibrium (LD) coefficients $\left(\left|D^{\prime}\right|\right.$ and $\left.r^{2}\right)$ among single nucleotide polymorphisms (SNPs) in the FOXJ1 gene

\begin{tabular}{|c|c|c|c|c|c|c|c|}
\hline SNP & \multicolumn{7}{|l|}{$\left|D^{\prime}\right|$} \\
\hline$g .-460 C>T$ & - & 1 & 0.060 & 0.386 & 0.386 & 0.386 & 1 \\
\hline$g .-342 G>C$ & 1 & - & 0.060 & 0.386 & 0.386 & 0.386 & 1 \\
\hline$g .1164 G>C$ & $5.0 \mathrm{E}-4$ & $5.0 \mathrm{E}-4$ & - & 1 & 1 & 1 & $3.4 \mathrm{E}-17$ \\
\hline g. $1805 G>T$ & 0.125 & 0.125 & 0.110 & - & 1 & 1 & 0.580 \\
\hline$g .3375 G>C$ & 0.858 & 0.858 & $1.9 \mathrm{E}-34$ & 0.243 & 0.243 & 0.243 & - \\
\hline
\end{tabular}

Table 3 Genotype and allele analysis of the polymorphisms in the FOXJ1 gene between allergic rhinitis patients and controls. $O R$ Odds ration, $C I$ confidence interval

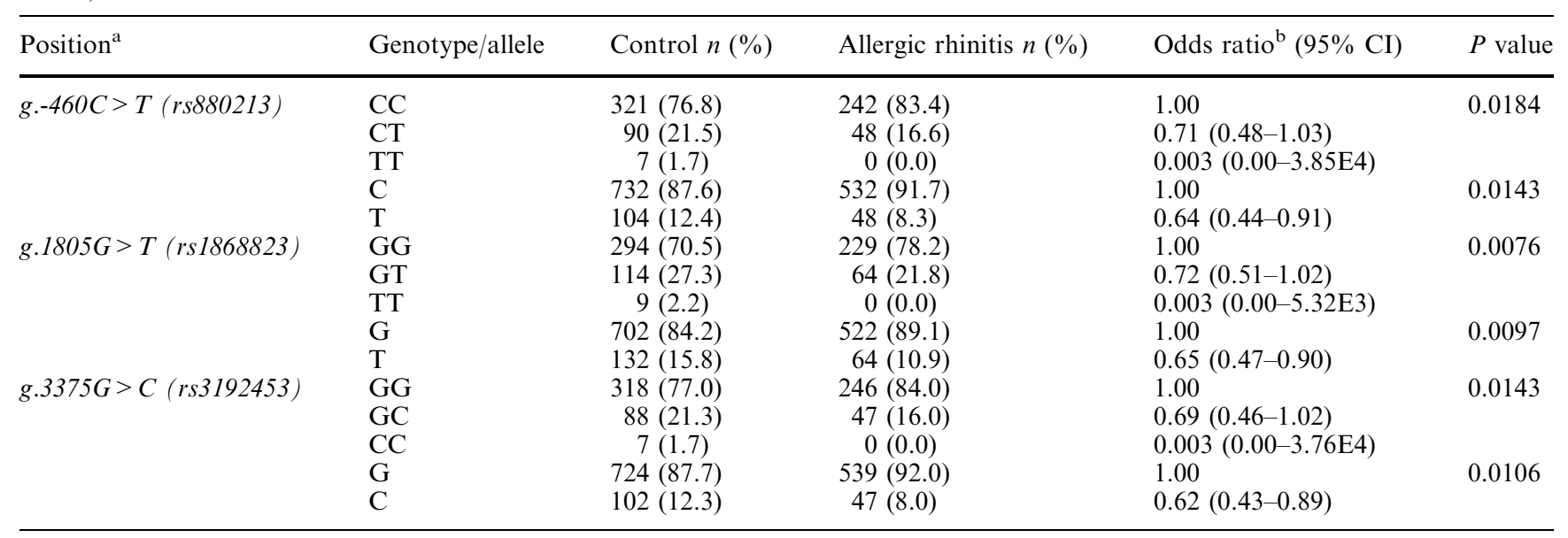

${ }^{\mathrm{a}}$ Calculated from the translation start site

${ }^{\mathrm{b}}$ Logistic regression analyses were used for calculating OR $(95 \% \mathrm{CI})$

factors. Several studies have reported analysis of the association of SNPs with allergic rhinitis. Cheng et al. (2004) reported that polymorphisms in disintegrin and the metalloprotease domain 33 (ADAM33) gene are associated with susceptibility to allergic rhinitis in the Japanese population. Polymorphisms of $I L-13$ were associated with allergic rhinitis susceptibility and might be responsible for the high IgE level in allergic rhinitis 
Table 4 Haplotype frequencies between allergic rhinitis patients and controls in FOXJ1 SNPs

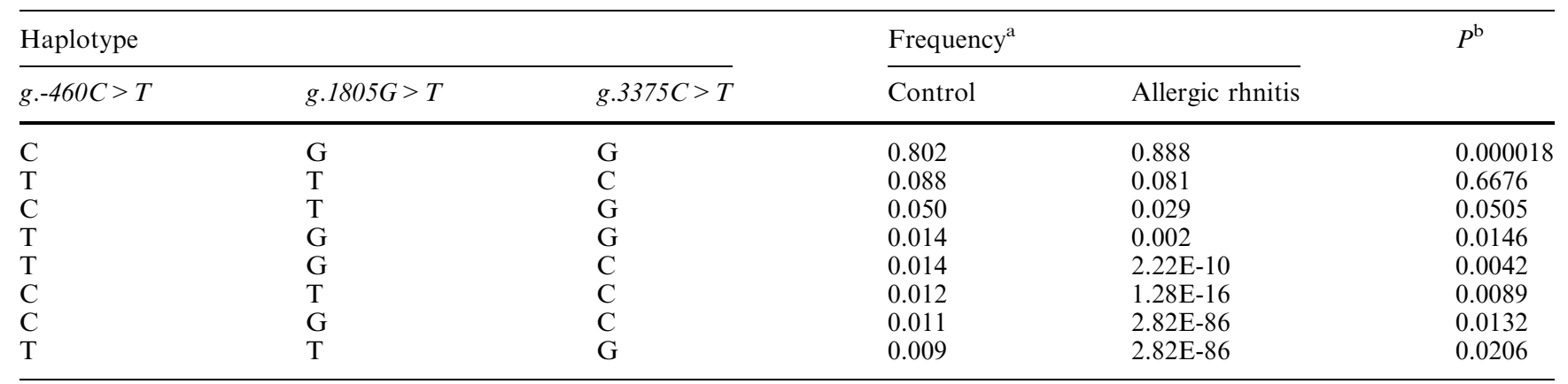

${ }^{a}$ Values were constructed using the expectation maximization (EM) algorithm with genotyped SNPs

${ }^{\mathrm{b}}$ Values were analyzed by permutation test

patients (Wang et al. 2003). We previously reported that polymorphisms of eotaxin-2, eotaxin-3 and the $\mathrm{T}$ cell immunoglobulin and mucin domain-3 (TIM-3) are associated with allergic rhinitis in the Korean population (Chae et al. 2004, 2005b). In our present study, FOXJ1 is identified as a novel gene associated with susceptibility to allergic rhinitis. Allergic rhinitis is characterized by predominance of Th2 cytokines and IgE production, and recruitment of mast cells, eosinophils and basophils to the site of allergic reactivity (Daser et al. 1995; O’Garra 1998). Naïve T helper cells can differentiate into Th1 or Th2 subsets. Th1 and Th2 cells can cross-regulate the differentiation of the partner subset. Foxjl plays a critical role in modulating inflammatory reactions and preventing autoimmunity by antagonizing pro-inflammatory transcriptional activities. Lin et al. (2004) observed that Foxj1-deficient mice suffer from systemic autoimmune inflammation, and that $\mathrm{CD}^{+}{ }^{+} \mathrm{T}$ cells isolated from Foxj1-deficient mice produce more IL-2 and IFN- $\gamma$ than wild type, indicating a skew towards a Th1-type response. The polymorphisms within the binding site of the promoter region might influence the expression level, by suppression of binding between the specific transcriptional binding site, and a transcription factor. Accordingly, it will be interesting to find out whether this polymorphism $(g .-460 C>T)$ might have some influence on susceptibility to allergic or autoimmune diseases. Thus, our results will be important in future studies, to determine whether or not this polymorphism affects the levels of FOXJI gene expression and function.

In summary, our results strongly suggest that the g.$460 C>T$, g. $1805 G>T$, and $g .3375 G>C$ polymorphisms in the FOXJ1 gene might be associated with susceptibility to allergic rhinitis, but not with IgE production. Our study is the first to analyze the association between $F O X J 1$ SNPs and allergic rhinitis, and may contribute to future studies on FOXJI function.

Acknowledgement This work was supported by a grant from the Korea Health 21 R\&D Project by Ministry of Health \& Welfare (01-PJ3-PG6-01GN09-003).

\section{References}

Blatt EN, Yan XH, Wuerffel MK, Hamilos DL, Brody SL (1999) Forkhead transcription factor HFH-4 expression is temporally related to ciliogenesis. Am J Respir Cell Mol Biol 21:168-176

Brody SL, Yan XH, Wuerffel MK, Song SK, Shapiro SD (2000) Ciliogenesis and left-right axis defects in forkhead factor $\mathrm{HFH}-$ 4-null mice. Am J Respir Cell Mol Biol 23:45-51

Carlsson P, Mahlapuu M (2002) Forkhead transcription factors: key players in development and metabolism. Dev Biol 250:1-23

Chae SC, Park YR, Lee YC, Lee JH, Chung HT (2004) The association of TIM-3 gene polymorphism with atopic disease in Korean population. Hum Immunol 65:1427-1431

Chae SC, Park YR, Oh GJ, Lee JH, Chung HT (2005a) The suggestive association of eotaxin-2 and eotaxin-3 gene polymorphisms in Korean population with allergic rhinitis. Immunogenetics 56:760-764

Chae SC, Park YR, Song JH, Shim SC, Yoon KS, Chung HT (2005b) The polymorphisms of Tim-1 promoter region are associated with rheumatoid arthritis in a Korean population. Immunogenetics 56:696-701

Chen J, Knowles HJ, Hebert JL, Hackett BP (1998) Mutation of the mouse hepatocyte nuclear factor/forkhead homologue 4 gene results in an absence of cilia and random left-right asymmetry. J Clin Invest 102:1077-1082

Cheng L, Enomoto T, Hirota T, Shimizu M, Takahashi N, Akahoshi M, Matsuda A, Dake Y, Doi S, Enomoto K, Yamasaki A, Fukuda S, Mao XQ, Hopkin JM, Tamari M, Shirakawa T (2004) Polymorphisms in ADAM33 are associated with allergic rhinitis due to Japanese cedar pollen. Clin Exp Allergy 34:1192-1201

Coffer PJ, Burgering BM (2004) Forkhead-box transcription factors and their role in the immune system. Nat Rev Immunol 4:889-899

Cookson W (2002) Genetics and genomics of asthma and allergic diseases. Immunol Rev 190:195-206

Daser A, Meissner N, Herz U, Renz H (1995) Role and modulation of T-cell cytokines in allergy. Curr Opin Immunol 7:762-770

Ho IC, Glimcher LH (2002) Transcription: tantalizing times for T cells. Cell 109:S109-S120

Kaestner KH, Knochel W, Martinez DE (2000) Unified nomenclature for the winged helix/forkhead transcription factors. Genes Dev 14:142-146

Lim L, Zhou H, Costa RH (1997) The winged helix transcription factor HFH-4 is expressed during choroid plexus epithelial development in the mouse embryo. Proc Natl Acad Sci USA 94:3094-3099

Lin L, Spoor MS, Gerth AJ, Brody SL, Peng SL (2004) Modulation of Th1 activation and inflammation by the NF-kappaB repressor Foxj1. Science 303:1017-1020 
Lundback B (1998) Epidemiology of rhinitis and asthma. Clin Exp Allergy 28:3-10

O'Garra A (1998) Cytokines induce the development of functionally heterogeneous $\mathrm{T}$ helper cell subsets. Immunity 8:275-283

Okada A, Ohta Y, Brody SL, Watanabe H, Krust A, Chambon P, Iguchi T (2004) Role of foxj1 and estrogen receptor alpha in ciliated epithelial cell differentiation of the neonatal oviduct. $\mathrm{J}$ Mol Endocrinol 32:615-625

Passali D, Lauriello M, Mezzedimi C, Passali GC, Bellussi L (2001) Natural history of allergic rhinitis: a review. Clin Appl Immunol Rev 1:207-216
Sibbald B (1993) Epidemiology of allergic rhinitis. Monogr Allergy 31:61-79

Tran H, Brunet A, Griffith EC, Greenberg ME (2003) The many forks in FOXO's road. Sci STKE 172: RE5

Wang M, Xing ZM, Lu C, Ma YX, Yu DL, Yan Z, Wang SW, Yu LS (2003) A common IL-13 Arg130Gln single nucleotide polymorphism among Chinese atopy patients with allergic rhinitis. Hum Genet 113:387-390

Weigel D, Jackle H (1990) The fork head domain: a novel DNA binding motif of eukaryotic transcription factors? Cell 63:455456 